\title{
Effect of the human papillomavirus (HPV) quadrivalent vaccine in a subgroup of women with cervical and vulvar disease: retrospective pooled analysis of trial data
}

\author{
(c) $(1)(9)$ OPEN ACCESS
}

Elmar A Joura associate professor ${ }^{1}$, Suzanne M Garland director, professor ${ }^{2}$, Jorma Paavonen professor, physician in chief ${ }^{3}$, Daron G Ferris professor ${ }^{4}$, Gonzalo Perez professor ${ }^{5}$, Kevin A Ault associate professor ${ }^{6}$, Warner K Huh associate professor ${ }^{7}$, Heather L Sings director of Global Scientific and Medical Publications ${ }^{8}$, Margaret K. James senior biometrician ${ }^{8}$, Richard M Haupt executive director of clinical research ${ }^{8}$, for the FUTURE I and II Study Group

${ }^{1}$ Department of Gynaecology and Obstetrics, Medical University of Vienna, Comprehensive Cancer Center, Währinger Gürtel 18-20, A-1090 Vienna, Austria; ${ }^{2}$ Microbiology and Infectious Diseases Department, Royal Women's Hospital and Department of Obstetrics and Gynaecology, University of Melbourne, Murdoch Childrens Research Institute, Melbourne, Victoria, Australia; ${ }^{3}$ Department of Obstetrics and Gynecology, University Central Hospital, Helsinki, Finland; ${ }^{4}$ Departments of Family Medicine and Obstetrics and Gynecology, Medical College of Georgia, Augusta, GA, USA; ${ }^{5}$ Universidad del Rosario, Bogotá, Colombia (now at Merck Sharp \& Dohme, Whitehouse Station); ${ }^{6}$ Department of Gynecology and Obstetrics and the Emory Vaccine Center, Emory University School of Medicine and Department of Global Health, Rollins School of Public Health, Emory University Atlanta GA, USA; ${ }^{7}$ Department of Obstetrics and Gynecology, University of Alabama at Birmingham, Birmingham, AL, USA; ${ }^{8}$ Merck Sharp \& Dohme, Whitehouse Station, NJ, USA

\begin{abstract}
Objectives To determine the effect of human papillomavirus (HPV) quadrivalent vaccine on the risk of developing subsequent disease after an excisional procedure for cervical intraepithelial neoplasia or diagnosis of genital warts, vulvar intraepithelial neoplasia, or vaginal intraepithelial neoplasia.

Design Retrospective analysis of data from two international, double blind, placebo controlled, randomised efficacy trials of quadrivalent HPV vaccine (protocol 013 (FUTURE I) and protocol 015 (FUTURE II)).

Setting Primary care centres and university or hospital associated health centres in 24 countries and territories around the world.

Participants Among 17622 women aged 15-26 years who underwent 1:1 randomisation to vaccine or placebo, 2054 received cervical surgery or were diagnosed with genital warts, vulvar intraepithelial neoplasia, or vaginal intraepithelial neoplasia.

Intervention Three doses of quadrivalent HPV vaccine or placebo at day 1 , month 2 , and month 6 .
\end{abstract}

Main outcome measures Incidence of HPV related disease from 60 days after treatment or diagnosis, expressed as the number of women with an end point per 100 person years at risk.

Results A total of 587 vaccine and 763 placebo recipients underwent cervical surgery. The incidence of any subsequent HPV related disease was 6.6 and 12.2 in vaccine and placebo recipients respectively (46.2\% reduction (95\% confidence interval $22.5 \%$ to $63.2 \%$ ) with vaccination). Vaccination was associated with a significant reduction in risk of any subsequent high grade disease of the cervix by $64.9 \%$ (20.1\% to $86.3 \%)$. A total of 229 vaccine recipients and 475 placebo recipients were diagnosed with genital warts, vulvar intraepithelial neoplasia, or vaginal intraepithelial neoplasia, and the incidence of any subsequent HPV related disease was 20.1 and 31.0 in vaccine and placebo recipients respectively (35.2\% reduction ( $13.8 \%$ to $51.8 \%)$ ).

Conclusions Previous vaccination with quadrivalent HPV vaccine among women who had surgical treatment for HPV related disease significantly reduced the incidence of subsequent HPV related disease, including high grade disease.

Trial registrations NCT00092521 and NCT00092534 


\section{Introduction}

The currently available human papillomavirus (HPV) vaccines that are based on recombinant virus-like particles are designed to prevent HPV associated disease. Both the quadrivalent vaccine (against HPV types 6,11, 16, and 18) and the bivalent vaccine (against types 16 and 18) are highly effective in preventing cervical intraepithelial neoplasia grade II-III or adenocarcinoma in situ in women who are not infected with the relevant HPV type before vaccination. ${ }^{1-5}$ The quadrivalent vaccine has also been shown to prevent HPV related vulvar intraepithelial neoplasia grade II-III, vaginal intraepithelial neoplasia grade II-III, and genital warts in women and to prevent genital warts and high grade anal disease in men. ${ }^{16-9}$

Current data show that HPV vaccination does not reduce progression to cervical precancers in women with ongoing infections at the time of vaccination. ${ }^{5}{ }^{10}{ }^{11}$ However, no studies to date have looked at the impact of HPV vaccination in preventing subsequent disease after treatment for such precancers. In two recent phase III clinical trials of the quadrivalent HPV vaccine, more than 17000 women were followed for approximately four years. Participants who underwent treatment for cervical, vulvar, or vaginal disease continued to be followed until the end of the study. We therefore had a unique opportunity to evaluate the post-treatment effect of vaccination in these women. In this retrospective analysis we identified women who had an excisional procedure for cervical disease or who were diagnosed with genital warts, vulvar intraepithelial neoplasia, or vaginal intraepithelial neoplasia after randomisation to vaccine or placebo and followed these women for additional disease outcomes. The objective of this study was to determine if the vaccine decreased the risk of developing subsequent disease after the first definitive treatment.

\section{Methods \\ Objectives}

The primary objective of this retrospective analysis was to determine whether administration of quadrivalent HPV vaccine, compared with placebo, reduced the incidence of subsequent HPV related disease among women who had undergone surgery for cervical disease or who were diagnosed with vulvar or vaginal disease (genital warts, vulvar intraepithelial neoplasia, or vaginal intraepithelial neoplasia) after enrolment into the FUTURE I or FUTURE II clinical trials (fig $1 \Downarrow$ ). Subsequent HPV related disease is defined as any disease that was detected at least 60 days after treatment or diagnosis. We measured the vaccine's impact on end points that were associated with HPV types $6,11,16$, and 18 specifically and on end points irrespective of causal HPV type.

\section{Study population}

Between December 2001 and May 2003, 17622 women aged 15-26 years were enrolled in one of two randomised, double blind, placebo controlled trials (FUTURE I and FUTURE II). ${ }^{12}$ The studies were conducted in accordance with principles of Good Clinical Practice and were approved by the appropriate institutional review boards and regulatory agencies.

The study designs and the results of the primary hypotheses have been described elsewhere. ${ }^{12}$ Women were eligible to participate if they were not pregnant, did not report previous abnormal results on a cervical smear test, and had had a lifetime number of no more than four sex partners. Neither study included HPV testing (DNA or serology testing) or clinical examination before randomisation; thus, the trials allowed the enrolment of women with ongoing HPV infection or disease.

\section{Intervention}

In both trials, participants were randomly assigned (1:1) to receive intramuscular injections of quadrivalent HPV vaccine (Gardasil or Silgard, Merck, Whitehouse Station, NJ) or visually indistinguishable placebo at day 1, month 2, and month 6 . Comprehensive anogenital examinations and ThinPrep cervical cytology (Cytyc, Boxborough, MA, USA) were performed during scheduled visits, which occurred every 6-12 months after the vaccination phase. ${ }^{12}$ Cytology specimens were classified according to the Bethesda System 2001. ${ }^{12}$ Women reported any abnormality or suspected disease in between the scheduled study visits. All participants were required to use birth control during the vaccination phase (day 1 through to month 7), and all participants were evaluated for pregnancy before the administration of each dose of vaccine or placebo with a human chorionic gonadotropin assay sensitive to 25 international units. ${ }^{13}$ Counselling about safe sex and contraceptive use was part of the study protocol.

Protocol specified guidelines were used to triage women with smear test abnormalities to colposcopy. ${ }^{12}$ Colposcopists were trained to locate and biopsy all discrete abnormal areas on the cervix and lower genital tract. Loop electrosurgical excision was the preferred method for definitive therapy for cervical intraepithelial neoplasia grade II-III, adenocarcinoma in situ, and persistent cervical intraepithelial neoplasia grade I. However, other methods included cervix conisation, cryotherapy, and electrocauterisation. The frequencies of each technique recorded were loop electrosurgical excision (84.7\%), cervical conisation $(12.5 \%)$, cryotherapy $(0.7 \%)$, and other $(2.1 \%)$. As loop electrosurgical excision and cervix conisation accounted for $97.2 \%$ of the procedures performed, with no difference between the treatment groups $(97.1 \%$ with placebo, $97.4 \%$ with vaccine) we use the term "cervical surgery" to describe any method used to treat cervical disease. Management of vulvar or vaginal disease followed the investigational site's standards and practice and included surgical or topical therapy.

\section{Population for retrospective subanalysis}

In this retrospective, intention to treat analysis, we identified all women who underwent definitive cervical therapy or who were diagnosed with vulvar or vaginal disease in the pooled FUTURE I and II studies after being randomised to and receiving at least one dose of HPV vaccine or placebo. The incidence rates for subsequent disease were calculated with case counting starting 60 days after cervical surgery or diagnosis of vulvar or vaginal disease.

\section{Rationale for 60 day window to define subsequent disease}

As stated previously, women were referred for colposcopy and cervical surgery in accordance with a triage algorithm. ${ }^{12}$ In FUTURE I, the pre-specified primary end points included all HPV related anogenital disease, thus the study included stringent criteria for disease determination and follow-up, including more frequent examinations and screening and more aggressive colposcopy triage for suspected disease. In FUTURE II, the primary end point was cervical intraepithelial neoplasia grade II-III and adenocarcinoma in situ. Treatment of vulvar or vaginal disease in FUTURE II was based on local standards of care, and the investigators were not required to record the date or method of treatment. We therefore examined the average time 
from diagnosis to treatment of genital warts, vulvar intraepithelial neoplasia, and vaginal intraepithelial neoplasia within the FUTURE I study. That information was used to determine an appropriate time frame after which most women would have been treated, so that follow-up after that window would probably capture new, rather than residual, disease. In FUTURE I, the mean number of days between diagnoses and treatment of genital warts, vulvar intraepithelial neoplasia, and vaginal intraepithelial neoplasia was 28 days (interquartile range 0-42 days). As the trials were less than four years of total duration (mean of 3.6 years), and since $82 \%$ had treatment within 60 days of the pathology panel diagnosis, we choose this as the cut off to ensure there would be enough follow-up time to detect subsequent disease after treatment. We assumed a similar time window between treatment and diagnosis in FUTURE II, and used the same window (60 days after diagnosis) for both studies. For consistency, the same time window was applied for counting subsequent disease after cervical surgery. A sensitivity analysis was also performed using a 90 day time window, as $91 \%$ of subjects had treatment within 90 days.

\section{Efficacy end points and statistics}

All specimens from biopsies and excisional procedures were tested for 14 HPV types $(6,11,16,18,31,33,35,39,45,51$, $52,56,58$, and 59) using an assay based on the polymerase chain reaction. ${ }^{14-16}$ All tissue specimens underwent histopathological review by a pathology panel, who were masked to the women's vaccination group and HPV status. A woman was considered to have developed an end point related to vaccine HPV types (HPV 6, 11, 16, or 18) or an end point related to 10 non-vaccine types (HPV 31, 33, 35, 39, 45, 51, $52,56,58$, or 59) if the respective HPV DNA were detected in the same lesion that was diagnosed by the pathology panel as cervical intraepithelial neoplasia, vulvar intraepithelial neoplasia, vaginal intraepithelial neoplasia, or genital warts. For analyses of all disease, irrespective of causal HPV type, a woman was considered to have developed an end point if she had a lesion diagnosed by the pathology panel without consideration of HPV status.

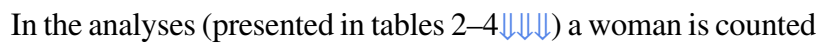
only once for each end point (that is, once in each row), but a woman may have developed more than one end point during the trial (that is, a women may appear in more than one row). For example, a woman who is diagnosed with a cervical intraepithelial grade 3 lesion that is positive to HPV types 16, 31 , and 45 would be counted once for (1) any disease; (2) any cervical disease; (3) any cervical intraepithelial grade II or worse; (4) any cervical intraepithelial grade III or worse; (5) any disease related to vaccine HPV types; (6) cervical intraepithelial grade I or worse related to vaccine HPV types; (7) cervical intraepithelial grade II or worse related to vaccine HPV types; and (8) cervical intraepithelial grade III or worse related to vaccine HPV types.

Women contributed to person years at risk from 60 days after the initial surgery or diagnosis until the day of ascertainment of the subsequent disease end point, or, for those without a subsequent disease end point, until the day of their last follow-up visit. Incidence, or rate of subsequent disease, is expressed as the number of women with an end point per 100 person years at risk. A point estimate of vaccine efficacy (that is, percentage reduction of the relative end point) and the $95 \%$ confidence interval were calculated on the basis of the observed case split between vaccine and placebo recipients, adjusted for the accrued person time in each group. The criterion for statistical significance $(\mathrm{P}<0.05)$ was equivalent to requiring that the lower bound of the confidence interval for vaccine efficacy exclude $0 \%$. An exact conditional procedure was used to evaluate vaccine efficacy under the assumption that the numbers of cases in the vaccine and placebo groups were independent Poisson random variables. ${ }^{17}$

Kaplan-Meier estimates (and 95\% confidence intervals) of cumulative incidence rates for subsequent disease were plotted with case counting starting 60 days after cervical surgery or vulvar or vaginal disease diagnosis. ${ }^{18}$ The plots were not part of a formal survival analysis; rather they give a visual demonstration of the divergence of the incidence rates between the two vaccination arms over time. The $95 \%$ confidence interval for the Kaplan-Meier incidence at intervals of six months are not directly comparable with vaccine efficacy estimates. Vaccine efficacy was calculated from the exact conditional procedure and is included in the Kaplan-Meier plots as a reference.

\section{Results}

\section{Analysis population}

The FUTURE I and II trials collectively enrolled and randomised 17622 women. The populations analysed to determine the impact of quadrivalent HPV vaccine on subsequent disease is shown in fig $2 \Downarrow$. Of the 1350 women included in the analysis, only five (three placebo recipients, two vaccine recipients) received fewer than all three doses, with one woman in the placebo group having only one dose, and the others receiving two doses each. These women stopped the vaccination phase because pregnancy (two women), lactation (one), medical history (one), and unknown reason (one), but continued the follow-up phase of the study.

As expected, more placebo recipients underwent cervical surgery for disease due to any HPV type $(n=763)$ than vaccine recipients $(\mathrm{n}=587)$. Among women who underwent cervical surgery, vaccine recipients had numerically higher baseline (at day 1 of the study) prevalence of squamous intraepithelial lesions (36.5\%) compared with placebo recipients $(30.0 \%)$ and higher prevalence of HPV DNA $(70.1 \%)$ than placebo $(62.0 \%)$ (table $1 \Downarrow$ ). Among women who had a diagnosis of genital warts, vulvar intraepithelial neoplasia, or vaginal intraepithelial neoplasia, vaccine recipients also had a higher baseline prevalence than placebo recipients of squamous intraepithelial lesions $(27.0 \%$ $v 14.2 \%)$ and HPV DNA $(65.9 \% v 44.0 \%)$ and a lower proportion of current or former smokers (36.7\% v 43.4\%).

\section{Incidence of subsequent disease in placebo group}

Placebo recipients who were treated for HPV related disease in these clinical trials were at increased risk for developing subsequent HPV related disease (fig $3 \Downarrow$ ). Within an average of only 1.3 years after cervical surgery (maximum follow-up of 3.7 years), the incidence of any subsequent disease among placebo recipients was 12.2 per 100 person years at risk, and $5.2 \%$ (31/593) developed subsequent high grade cervical, vulvar, or vaginal disease (incidence of 3.7). Compared with those who underwent cervical surgery, those who were diagnosed with genital warts, vulvar intraepithelial neoplasia, or vaginal intraepithelial neoplasia had nearly three times the risk for developing any subsequent HPV related disease (incidence of 31.0). Within an average of only 1.2 years after the initial diagnosis of vulvar or vaginal disease (maximum follow-up of 4.0 years), $13.0 \%$ (55/422) developed high grade cervical, vulvar, or vaginal disease (incidence of 8.4). For both analysis populations, the incidence of subsequent low grade disease 
(genital warts; vulvar or vaginal or intraepithelial neoplasia grade I; or cervical intraepithelial neoplasia grade I) was also high (10.1 to 26.1).

\section{Impact of prior vaccination on disease after cervical surgery}

Vaccination was associated with a significantly reduced risk of any subsequent HPV related disease after cervical surgery, irrespective of causal HPV type, by $46.2 \%$ (95\% confidence interval $22.5 \%$ to $63.2 \%$ ) (table $2 \Downarrow$ ). Vaccination was associated with a significantly reduced risk of any subsequent cervical disease (by $48.3 \%$ (95\% confidence interval $19.1 \%$ to $67.6 \%$ ) for cervical intraepithelial neoplasia grade I or worse) and any subsequent high grade cervical disease (64.9\% (20.1\% to $86.3 \%)$ for cervical intraepithelial neoplasia grade II or worse). A significant reduction in the incidence of genital warts was observed $(63.0 \%$ (10.3\% to $86.6 \%)$ ). Vaccination was also associated with significantly reduced risk of any subsequent disease related to vaccine HPV types (79.1\% (49.4\% to 92.8\%)). The impact on vulvar or vaginal disease was primarily driven by a reduction in the incidence of genital warts. Vaccine recipients saw an $89.0 \%$ reduction $(54.9 \%$ to $98.7 \%$ ) in genital warts related to vaccine HPV types after cervical surgery. Vaccine efficacy estimates were similar in the sensitivity analysis which used a 90 day interval between the first and subsequent disease diagnoses (see supplementary table 1 on bmj.com).

As shown in table $2 \Downarrow$, two vaccine and nine placebo recipients developed cervical disease related to vaccine HPV types after their first cervical surgery. Of the two women in the vaccine arm, one developed cervical intraepithelial neoplasia grade I and II lesions related to HPV type 16 after surgery. This woman was DNA positive to HPV types $16,31,56$, and 58 at study entry and had HPV types 16 and 58 detected in her original surgical specimen. The second woman developed a cervical intraepithelial neoplasia grade I lesion related to HPV 18. She was DNA positive to HPV 18 at day 1 (and was negative to the other 11 tested high risk HPV types) but underwent electrocauterisation, so no surgical specimen existed. Of the nine women in the placebo arm who developed cervical disease related to vaccine HPV types, six $(67 \%)$ did not have the same vaccine HPV type detected in their original surgical specimen.

Table $3 \Downarrow$ shows the vaccine efficacy for cervical intraepithelial neoplasia grade I or worse due to 10 high risk, non-vaccine HPV types after surgery for cervical disease. Vaccination was associated with reduction of cervical intraepithelial neoplasia grade I or worse associated with HPV31, 33, 45, 52, 58 (the five most common HPV types found in cervical cancer after HPV16 and HPV18) by $56.6 \%$ (3.4\% to $82.3 \%$ ). For individual HPV types, a significant reduction was observed for HPV31 (88.4\%, [18.6\% to $99.7 \%]$ ). A positive percent reduction was observed for seven of the other types analysed, though the data did not reach statistical significance.

Fig $4 \Downarrow$ shows the divergence of incidence rates over time for any subsequent disease and any subsequent vulvar or vaginal disease, irrespective of causal HPV type. Among both vaccine and placebo recipients, the incidence of any subsequent HPV related disease (fig 4A) had not reached a plateau by the end of study, and the divergence of incidence between the vaccine group and the placebo group increased at each six month interval. In contrast, the incidence of subsequent vulvar or vaginal disease after cervical surgery seemed to have reached a plateau for both vaccine and placebo recipients by end of study (fig 4B).

\section{Impact of prior vaccination on disease after diagnosis of genital warts or of vulvar or vaginal intraepithelial neoplasia}

Vaccination was associated with a reduced risk of any subsequent HPV related disease, irrespective of causal HPV type, by $35.2 \%$ ( $13.8 \%$ to $51.8 \%$ ) (table $4 \Downarrow$ ). For end points related to vaccine HPV types, there was a $64.4 \%$ reduction $(41.6 \%$ to $79.3 \%)$ in any disease observed. Fig $4 \Downarrow$ shows the divergence of incidence rates over time for any subsequent disease, and any subsequent cervical disease, irrespective of causal HPV type. Among both vaccine and placebo groups, the incidence of any subsequent disease (fig 4C) and any subsequent cervical disease (fig 4D) had not reached a plateau by end of study. Vaccine efficacy estimates were similar in the sensitivity analysis which used a 90 day interval between initial surgery or disease diagnosis and subsequent disease diagnoses (supplementary table 2 on bmj.com).

\section{Impact of prior vaccination on recurrent genital warts and low grade disease}

In the previous analyses, we considered the impact of vaccination collectively among women who were diagnosed with genital warts, vulvar intraepithelial neoplasia grade I or worse, or vaginal intraepithelial neoplasia grade I or worse. If we consider only those women who were diagnosed with genital warts, there were 134 vaccine and 351 placebo recipients, respectively. The average time to the first detection of genital warts in these women was 1.4 years in the vaccine group and 2.1 years in the placebo group. Of these women, $43.3 \%$ (58/134) vaccine recipients and 14.8\% (52/351) placebo recipients were infected with HPV types 6 or 11 at study entry. When we followed these women for recurrent genital warts, vaccination was associated with less recurrence of genital warts related to vaccine HPV types by $46.8 \%$ (10 cases in vaccine group $v 33$ cases in placebo group), but the reduction was not statistically significant.

A similar analysis was performed for recurrent vulvar or vaginal low grade disease (genital warts, vulvar intraepithelial neoplasia grade I, or vaginal intraepithelial neoplasia grade I). There were 210 vaccine recipients and 445 placebo recipients who were diagnosed with low grade disease. Vaccination was associated with reduced risk of subsequent low grade disease related to vaccine HPV types by $60.3 \%$ ( $21.7 \%$ to $81.5 \%)$.

\section{Discussion}

Our results of the subgroup analysis demonstrate that vaccination with the quadrivalent HPV vaccine was associated with reduced incidence of subsequent cervical, vulvar, and vaginal intraepithelial neoplasia and genital warts in women who had been diagnosed and treated for cervical and vulvar or vaginal disease. After treatment for high grade cervical intraepithelial neoplasia, women are at increased risk for cervical cancer in the long term, ${ }^{19}$ and our data show they are at risk for subsequent diagnoses of premalignant disease in the short term. Within two years after treatment, those with prior vaccination had significantly reduced risk of subsequent high grade cervical disease. Our analysis also shows that for women who were diagnosed with HPV related vulvar or vaginal disease, those with prior vaccination had significantly reduced risk of developing any subsequent HPV related disease as well as disease related to the vaccine HPV types (types 6, 11, 16, and 18 ). These are, to our knowledge, the first results in vaccinated women who have undergone treatment for HPV related diseases. 


\section{Strengths of study}

We took several steps to enhance the accuracy, reproducibility, and generalisability of our findings. We considered all disease end points, irrespective of causal HPV type. Histological diagnoses were determined by a panel of expert gynaecologic pathologists who were unaware of women's treatment or history of cervical or vulvar disease. Generalisability was enhanced by enrolling women from both developed and developing nations and by using standard management algorithms for treatment of cervical disease and local standards of care for treatment of vulvar or vaginal disease..$^{20}$

In addition to measuring the impact on precancerous lesions, we also measured the impact on low grade disease. Although low grade cervical and vulvar or vaginal lesions are considered to be a morphological manifestation of an HPV infection, these lesions contribute to the clinical and economic burden of HPV disease. For example, the psychosocial ${ }^{22-24}$ and economic ${ }^{25} 26$ implications of genital warts are substantial and reflect, in part, the high transmission and recurrence rates. ${ }^{27-29}$ Lesion and infection are a local phenomenon of the transformation zone at the cervix, which is completely removed by treatment, whereas vulvovaginal disease is a field infection (infectious areas are not completely removed by the treatment and, therefore, the recurrence rate is high). In contrast to breast and cervical cancers, there are no screening programmes for vaginal and vulvar malignancies, and precursors of vulvar and vaginal cancers are often not recognised. ${ }^{7}$ Cervical, vulvar, and vaginal intraepithelial neoplasia grade I and genital warts have also been shown to contain a variety of high risk HPV types. ${ }^{60-33}$ In this study, the highest rates of subsequent disease observed was low grade, and vaccination rapidly and significantly reduced the onset of subsequent low grade lesions, particularly among women who underwent cervical surgery.

\section{Comparison with other studies}

These data are in line with previous studies that suggest the benefits of vaccination are not limited to the primary target group of young, sexually naïve girls. In an intention to treat analysis that measured the impact of the quadrivalent HPV vaccine in a mixed population of HPV naïve and HPV infected women, vaccination significantly reduced the number of abnormal smear test results and procedures such as colposcopy, biopsy examination, and definitive therapy irrespective of the causal HPV type. ${ }^{34}$ Another study has shown the quadrivalent HPV vaccine generates an anamnestic response (renewed rapid production of an antibody on a subsequent encounter with the same antigen) in women aged 15-26 who are seropositive before vaccination, ${ }^{35}{ }^{36}$ and that the quadrivalent HPV vaccine prevents reinfection or reactivation of disease that is related to vaccine HPV types. ${ }^{37}$ For example, women who had cleared an HPV16 infection in the past were protected from developing subsequent HPV16 related disease.$^{37}$ A previous study has shown that women who are infected with one or more vaccine HPV types derive residual benefit by the quadrivalent HPV vaccine's prevention of infection and disease from HPV type(s) to which the woman has not yet been exposed. ${ }^{38}$ The vaccine is also effective in women up to the age of 45 , whereby prophylactic vaccine efficacy against disease related to vaccine HPV types was $92.4 \%$ (49.6\% to 99.8$).{ }^{39} 40$

\section{Limitations of study}

This retrospective analysis is accompanied by some limitations. The trials were not designed or powered to evaluate the effects of vaccination after cervical surgery or diagnosis. Women with a prior history of HPV related disease were excluded from enrolment, so we cannot directly measure the vaccine's impact in women who have undergone treatment before vaccination, since all women in this study were vaccinated before treatment. As a surrogate, we identified women who were treated for HPV related disease after randomisation and followed them for additional disease outcomes. In this relatively low risk population (healthy women with a mean of two lifetime sexual partners and no prior history of HPV related disease) the risk of subsequent disease after a diagnosis of HPV related vulvar or vaginal disease was about three times higher than after cervical surgery. Although the reasons for this are unclear, our data indicate that women who were diagnosed with HPV related disease were a high risk group at study entry, as nearly half of these women (44-70\%) were infected with at least one of the 14 tested HPV types, and 14-37\% had abnormal cytology at day 1 .

There was an imbalance in some baseline characteristics (that is, at day 1) as a result of our selection of patients who had treatment for cervical or vulvovaginal disease after vaccination that may have potentially biased these results. Among women who had a pathology panel diagnosis of vulvar or vaginal disease, placebo recipients had a higher proportion of current or former smokers (43.4\%) compared with vaccine recipients $(36.7 \%)$, and women who smoke are about twice as likely as non-smokers to get cervical cancer. ${ }^{41}$ However, the most important risk factor for cervical cancer is infection by HPV, ${ }^{41}$ and, because of our subsetting to those who later received treatment for cervical or vulvovaginal disease, vaccine recipients had numerically higher day 1 prevalence of HPV DNA and day 1 prevalence of squamous intraepithelial lesions compared with placebo recipients. In spite of these potential biases, there were still significant reductions in subsequent disease for vaccine recipients compared with placebo recipients.

It is important to note that most of the "first" disease detected in both vaccine and placebo recipients was a result of these infections that were present at day 1 , and not due to vaccine failure. It is also important to note that the impact of the vaccine in preventing subsequent disease does not indicate that vaccination provides a therapeutic effect. Though it is possible that some of the cervical disease detected after cervical definitive therapy is residual, these data suggest that most of the subsequent disease is from an infection with an HPV type that was not the causal type of the first lesion. For example, we found that six of the nine women in the placebo arm who developed cervical disease related to vaccine HPV types after surgery had a vaccine HPV type that was not detected in the original surgical specimen, suggesting that the lesion diagnosed after surgery was from a new infection and not from residual disease. Four of the six women in the placebo group who developed cervical intraepithelial neoplasia related to a vaccine HPV type after surgery that was not in the original surgical specimen also reported at least one new sexual partner in the interval between the first cervical surgery and the subsequent disease diagnosis. We have also previously shown that vaccination of women who were negative to 14 HPV types at enrolment reduced the incidence of cervical intraepithelial neoplasia grade III or worse associated with 10 non-vaccine HPV types by $32.5 \%$ (6.0\% to $51.9 \%)$, and the present analyses suggest some of the subsequent disease that is prevented is due to protection against HPV types that are phylogenetically related to vaccine HPV types $\left(6,11,16\right.$, and 18). ${ }^{42}$ Although the exact underlying mechanism is not fully known, these observed reductions are felt to be clinically significant. 


\section{Conclusions}

At present, in the United States and other countries, HPV testing before HPV vaccination is not recommended..$^{43}$ Data to date indicate HPV vaccination does not reduce progression to cervical pre-cancers in women with ongoing infection, and cervical cancer screening and corresponding management should continue as per local guidelines. ${ }^{510} 11$ Our study confirms that vaccination does not reduce progression to disease in women who are infected with HPV at the time of vaccination, but women who were treated for disease in the context of these studies were at risk for developing subsequent disease, and vaccination offered substantial benefit. Prophylactic HPV vaccines have been shown to remain efficacious for at least 10 years, ${ }^{44}$ and so it can be anticipated that vaccinated women will continue to benefit from a reduction in disease risk in the long term. However, only long term surveillance of vaccinated populations can determine the population effectiveness of vaccination. Several programmes to monitor both the safety and the impact of HPV vaccines on disease outcomes are in place. ${ }^{45} 46$

We thank Kathy Harkins, Carolyn Maass, MaryAnne Rutkowski, and Shuang Lu for statistical and programming support and Karyn Davis for publication support. We also thank all study staff and participants.

Contributors: This retrospective analysis was designed by external investigators in collaboration with the sponsor (Merck). The sponsor performed the statistical analysis. The authors were actively involved in the collection, analysis, or interpretation of the data, revising the manuscript for intellectual content, and approval of the final manuscript. All authors had full access to the data and had final responsibility for the decision to submit for publication. EAJ assumes full responsibility for the overall content and integrity of the manuscript.

Funding: The study was funded by Merck, Sharp, \& Dohme, Whitehouse Station.

Competing interests: All authors have completed the Unified Competing Interest form at www.icmje.org/coi_disclosure.pdf (available on request from the corresponding author) and declare that EAJ has received advisory board fees from Merck, and funding through his institution to conduct epidemiological HPV studies for GlaxoSmithKline. He has received lecture fees from Merck, Sanofi Pasteur MSD, and GlaxoSmithKline. SMG has received advisory board fees and grant support from Commonwealth Serum Laboratories and GlaxoSmithKline, and lecture fees from Merck and Sanofi Pasteur MSD. She has received funding through her institutions to conduct HPV vaccine studies for GlaxoSmithKline. DGF has received consultancy fees and funding through his institution to conduct HPV vaccine studies for GlaxoSmithKline, and lecture fees, advisory board fees, and consulting from Merck. GP has received lecture fees from Merck and Sanofi Pasteur MSD and is now an employee of Merck. KAA has received consultancy and advisory board fees from Merck, and has received funding through his institution to conduct HPV vaccine studies for GlaxoSmithKline, Roche, and the National Institute for Allergy and Infectious Diseases, and for non-vaccine clinical trials for Gen-Probe. WKH has received advisory board and lecture fees from Merck. He has received advisory board fee from GlaxoSmithKline. In addition, EAJ, SMG, DGF JP, KA, and WKH have received funding through their institutions to conduct HPV vaccine studies for Merck. HLS, MKJ, GP, and RMH and are employees of Merck and potentially own stock or stock options in the company.

Ethical approval: Studies were conducted in conformity with country or local requirements regarding ethics committee review, informed consent, and other statutes or regulations regarding the rights and welfare of human subjects participating in biomedical research.

Data sharing: No additional data available.
1 Garland SM, Hernandez-Avila M, Wheeler CM, Perez G, Harper DM, Leodolter S, et al. Quadrivalent vaccine against human papillomavirus to prevent anogenital diseases. $N$ Engl J Med 2007;356:1928-43.

2 The FUTURE II Study Group. Quadrivalent vaccine against human papillomavirus to prevent high-grade cervical lesions. N Engl J Med 2007;356:1915-27.

3 The FUTURE II Study Group. Effect of prophylactic human papillomavirus L1 virus-like-particle vaccine on risk of cervical intraepithelial neoplasia grade 2 , grade 3 and adenocarcinoma in situ: a combined analysis of four randomised clinical trials. Lancet 2007;369:1861-8.

4 Paavonen J, Jenkins D, Bosch FX, Naud P, Salmeron J, Wheeler CM, et al. Efficacy of a prophylactic adjuvanted bivalent $L 1$ virus-like-particle vaccine against infection with human papillomavirus types 16 and 18 in young women: an interim analysis of a phase III double-blind, randomised controlled trial. Lancet 2007;369:2161-70.

5 Paavonen J, Naud P, Salmeron J, Wheeler CM, Chow SN, Apter D, et al. Efficacy of human papillomavirus (HPV)-16/18 AS04-adjuvanted vaccine against cervical infection and precancer caused by oncogenic HPV types (PATRICIA): final analysis of a double-blind, randomised study in young women. Lancet 2009;374:301-14.

6 Garland SM, Steben M, Sings HL, James M, Lu S, Railkar R, et al. Natural history of genital warts: analysis of the placebo arm of 2 randomized phase III trials of a quadrivalent human papillomavirus (types 6, 11, 16, and 18) vaccine. J Infect Dis 2009;199:805-14.

7 Joura EA, Leodolter S, Hernandez-Avila M, Wheeler CM, Perez G, Koutsky LA, et al. Efficacy of a quadrivalent prophylactic human papillomavirus (types 6, 11, 16 and 18) L1 virus-like-particle vaccine against high-grade vulval and vaginal lesions: a combined analysis of three clinical trials. Lancet 2007;369:1693-702.

8 Merck Research Laboratories.GARDASIL® (human papillomavirus [types 6, 11, 16, 18] recombinant vaccine) supplemental biologics licensing application for use in anal cancer prevention. Vaccines and Related Biological Products Advisory Committee (VRBPAC) prevention. Vaccines and Related Biological Products Advisory Committee/
briefing document. 2010. www.fda.gov/downloads/AdvisoryCommittees/ CommitteesMeetingMaterials/BloodVaccinesandOtherBiologics/ VaccinesandRelatedBiologicalProductsAdvisoryCommittee/UCM231522.pdf.

9 Palefsky JM, Giuliano AR, Goldstone S, Moreira ED, Aranda C, Jessen H, et al. HPV vaccine against anal HPV infection and anal intraepithelial neoplasia. N Engl J Med 2011;365:1576-85.

10 Kapeu AS, Luostarinen T, Jellum E, Dillner J, Hakama M, Koskela P, et al. Is smoking an independent risk factor for invasive cervical cancer? A nested case-control study within Nordic biobanks. Am J Epidemiol 2009;169:480-8.

11 Haupt RM, Wheeler CM, Brown DR, Garland SM, Ferris DG, Paavonen JA, et al. Impact of an HPV6/11/16/18 L1 virus-like particle vaccine on progression to cervical intraepithelial neoplasia in seropositive women with HPV16/18 infection. Int J Cancer 2011:129:2632-42.

12 Henry MR. The Bethesda system 2001: an update of new terminology for gynecologic cytology. Clin Lab Med 2003;23:585-603.

13 Garland SM, Ault KA, Gall SA, Paavonen J, Sings HL, Ciprero KL, et al. Pregnancy and infant outcomes in the clinical trials of a human papillomavirus type 6/11/16/18 vaccine: a combined analysis of five randomized controlled trials. Obstet Gynecol 2009;114:1179-88.

14 International patent numbers WO 2003/019143 A2, WO 2006/116276 A2, and WO 2006/116303 A2. 2008. www.wipo.int/portal/index.html.en.

15 Villa LL, Costa RLR, Petta CA, Andrade RP, Ault KA, Giuliano AR, et al. Prophylactic quadrivalent human papillomavirus (types $6,11,16$ and 18 ) L1 virus-like particle vaccine in young women: a randomised double-blind placebo-controlled multicentre phase II efficacy trial. Lancet Oncol 2005;6:271-8.

16 Mao C, Koutsky LA, Ault KA, Wheeler CM, Brown DR, Wiley DJ, et al. Efficacy of human papillomavirus-16 vaccine to prevent cervical intraepithelial neoplasia: a randomized controlled trial. Obstet Gynecol 2006;107:18-27.

17 Chan ISF, Bohidar NR. Exact power and sample size for vaccine efficacy studies. Commun Stat Theory Meth 1998;27:1305-22.

18 Kaplan EL, Meier P. Nonparametric estimation from incomplete observations. J Am Stat Assoc 1958;53:457-81.

19 McCredie MR, Sharples KJ, Paul C, Baranyai J, Medley G, Jones RW, et al. Natural history of cervical neoplasia and risk of invasive cancer in women with cervical intraepithelial neoplasia 3: a retrospective cohort study. Lancet Oncol 2008;9:425-34.

20 Wright TJ, Massad L, Dunton C, Spitzer M, Wilkinson E, Solomon D. 2006 consensus guidelines for the management of women with cervical intraepithelial neoplasia or adenocarcinoma in situ. J Low Genit Tract Dis 2007;11:223-39.

21 Ferris D. The 2001 ASCCP management guidelines for cervical cytology. Am Fam Physician 2004;70:1866-8

22 Clarke P, Ebel C, Catotti DN, Stewart S. The psychosocial impact of human papillomavirus infection: implications for health care providers. Int J STD AIDS 1996;7:197-200.

23 Maw RD, Reitano M, Roy M. An international survey of patients with genital warts: perceptions regarding treatment and impact on lifestyle. Int J STD AIDS 1998;9:571-8.

24 The FUTURE II Study Group. Four year efficacy of prophylactic human papillomavirus (types 6, 11, 16, and 18) L1 virus-like particle vaccine against low-grade cervical, vulvar, and vaginal intraepithelial neoplasia and condylomata acuminata. BMJ 2010;340:1-9.

25 Insinga RP, Dasbach EJ, Myers ER. The health and economic burden of genital warts in a set of private health plans in the United States. Clin Infect Dis 2003;36:1397-403.

26 Langley PC, White DJ, Drake SM. The costs of treating external genital warts in England and Wales: a treatment pattern analysis. Int J STD AIDS 2004;15:501-8.

27 Kodner CM, Nasraty S. Management of genital warts. Am Fam Physician 2004;70:2335-42.

28 Lacey CJ. Therapy for genital human papillomavirus-related disease. J Clin Virol 2005;32:S82-90

29 Wiley DJ, Douglas J, Beutner K, Cox T, Fife K, Moscicki AB, et al. External genital warts: diagnosis, treatment and prevention. Clin Infect Dis 2002;35:S210-24.

30 Clifford GM, Rana RK, Franceschi S, Smith JS, Gough G, Pimenta JM. Human papillomavirus genotype distribution in low-grade cervical lesions: comparison by geographic region and with cervical cancer. Cancer Epidemiol Biomarkers Prev 2005;14:1157-64.

31 Herrero R, Castle PE, Schiffman M, Bratti MC, Hildesheim A, Morales J, et al. Epidemiologic profile of type-specific human papillomavirus infection and cervical neoplasia in Guanacaste, Costa Rica. J Infect Dis 2005;191:1796-807.

32 Srodon M, Stoler MH, Baber GB, Kurman RJ. The distribution of low and high-risk HPV types in vulvar and vaginal intraepithelial neoplasia (VIN and VaIN). Am J Surg Pathol 2006;30:1513-8.

33 Garland SM, Insinga RP, Sings HL, Haupt RM, Joura EA. Human papillomavirus infections and vulvar disease development. Cancer Epidemiol Biomarkers Prev 2009;18:1777-84. 


\section{What is already known on this subject}

Prophylactic vaccination with quadrivalent HPV vaccine is highly efficacious in preventing cervical intraepithelial neoplasia, adenocarcinoma in situ, vulvar intraepithelial neoplasia, vaginal intraepithelial neoplasia, and genital warts

HPV vaccination does not reduce progression to cervical pre-cancers in women with ongoing infections at the time of vaccination, but the effect of vaccination in preventing subsequent disease after treatment is unknown

\section{What this study adds}

Vaccination with quadrivalent HPV vaccine was associated with a reduction of subsequent cervical, vulvar, and vaginal intraepithelial neoplasia and genital warts in women who were diagnosed and treated for cervical and vulvar or vaginal disease in two clinical trials of four year duration

Vaccination was associated with a reduction of subsequent disease irrespective of causal HPV type by $35-46 \%$

34 Munoz N, Kjaer SK, Sigurdsson K, Iversen O-E, Hernandez-Avila M, Wheeler CM, et al. Impact of human papillomavirus (HPV)-6/11/16/18 vaccine on all $\mathrm{HPV}$-associated genital diseases in young women. J Natl Cancer Inst 2010;102:325-39.

35 Villa LL, Ault K, Giuliano AR, Costa RLR, Petta CA, Andrade RP, et al. Immunologic responses following administration of a vaccine targeting human papillomavirus types 6 11,16 and 18. Vaccine 2006;24:5571-83

36 Olsson S-E, Villa LL, Costa R, Petta C, Andrade R, Malm C, et al. Induction of immune memory following administration of a prophylactic quadrivalent human papillomavirus (HPV) types 6/11/16/18 L1 virus-like-particle vaccine. Vaccine 2007;25:4931-9.

37 Olsson S-E, Kjaer SK, Sigurdsson K, Iversen O-E, Hernandez-Avila M, Wheeler CM, et al. Evaluation of quadrivalent HPV 6/11/16/18 vaccine efficacy against cervical and anogenital disease in subjects with serological evidence of prior vaccine type HPV infection. Human Vaccines 2009;5:696-704.

38 The FUTURE II Study Group. Prophylactic efficacy of a quadrivalent human papillomavirus (HPV) vaccine in women with virologic evidence of HPV infection. J Infect Dis 2007; $196: 1438-46$

39 Munoz N, Manalastas R, Pitisuttithum P, Tresukosol D, Monsonego J, Ault K, et al. Safety, immunogenicity, and efficacy of quadrivalent HPV (types $6,11,16,18$ ) recombinant vaccine in adult women between 24 and 45 years of age: a randomized, double-blind trial. Lancet 2009;373:1949-57.

40 Castellsague X, Munoz N, Pitisuttithum P, Ferris D, Monsonego J, Ault K, et al. End-of-study safety, immunogenicity, and efficacy of quadrivalent HPV (types 6, 11, 16, 18) recombinant vaccine in adult women 24-45 years of age. Br J Cancer 2011;105:28-37.

41 American Cancer Society. What are the risk factors for cervical cancer? 2011. www. cancer.org/Cancer/CervicalCancer/DetailedGuide/cervical-cancer-risk-factors.
42 Brown DR, Kjaer SK, Sigurdsson K, Iversen O-E, Hernandez-Avila M, Wheeler CM, et al. The impact of quadrivalent HPV (type 6/11/16/18) L1 VLP vaccine on infection and disease due to oncogenic non-vaccine HPV types in generally HPV naive women aged 16-26. J Infect Dis 2009;199:926-35.

43 Saslow D, Castle PE, Cox JT, Davey DD, Einstein MH, Ferris DG, et al. American Cancer Society Guideline for human papillomavirus (HPV) vaccine use to prevent cervical cancer and its precursors. CA Cancer J Clin 2007;57:7-28.

44 Rowhani-Rahbar A, Mao C, Hughes JP, Alvarez FB, Bryan JT, Hawes SE, et al. Long-term efficacy of a prophylactic human papillomavirus type 16 vaccine. Vaccine2009:27:5612-9.

45 Markowitz LE, Hariri S, Unger ER, Saraiya M, Datta SD, Dunne EF. Post-licensure monitoring of HPV vaccine in the United States. Vaccine 2010;28:4731-7.

46 Bonanni P, Cohet C, Kjaer SK, Latham NB, Lambert PH, Reisinger K, et al. A summary of the post-licensure surveillance initiatives for GARDASIL/SILGARD(R). Vaccine 2010;28:4719-30.

Accepted: 13 January 2012

\section{Cite this as: BMJ 2012;344:e1401}

This is an open-access article distributed under the terms of the Creative Commons Attribution Non-commercial License, which permits use, distribution, and reproduction in any medium, provided the original work is properly cited, the use is non commercial and is otherwise in compliance with the license. See: $\mathrm{http}: / /$ creativecommons.org/licenses/bync/2.0/ and http://creativecommons.org/licenses/by-nc/2.0/legalcode. 


\section{Tables}

Table 1/ Characteristics at study enrolment (day 1 ) of women aged 15-26 years who had undergone cervical surgery or had a diagnosis of vulvar or vaginal disease ${ }^{\star}$ after randomisation to quadrivalent HPV vaccine or placebo. Values are numbers (percentages) unless stated otherwise

Women who had cervical surgery Vaccine $(\mathrm{n}=587)$ Placebo $(\mathrm{n}=763)$

Mean (SD) age (years)

Race or ethnicity:

\begin{tabular}{|c|c|c|c|c|}
\hline Asian & $15(2.6)$ & $18(2.4)$ & $6(2.6)$ & $14(2.9)$ \\
\hline Black & $23(3.9)$ & $41(5.4)$ & $7(3.1)$ & $33(6.9)$ \\
\hline Hispanic American & $76(12.9)$ & $110(14.4)$ & $49(21.4)$ & $88(18.5)$ \\
\hline Native American & 0 & $1(0.1)$ & 0 & $1(0.2)$ \\
\hline White & $407(69.3)$ & $520(68.2)$ & $136(59.4)$ & $304(64.0)$ \\
\hline Other & $66(11.2)$ & $73(9.6)$ & $31(13.5)$ & $35(7.4)$ \\
\hline \multicolumn{5}{|l|}{ Smoking status: } \\
\hline Current & $187(31.9)$ & $260(34.1)$ & $64(27.9)$ & $177(37.3)$ \\
\hline Former & $45(7.7)$ & $46(6.0)$ & $20(8.7)$ & $29(6.1)$ \\
\hline Never & $355(60.5)$ & $457(59.9)$ & $145(63.3)$ & $269(56.6)$ \\
\hline Virgins & $9(1.5)$ & $20(2.6)$ & $4(1.7)$ & $23(4.8)$ \\
\hline \multicolumn{5}{|l|}{ Non-virgins' lifetime No of sexual partners: } \\
\hline 1 & $117 / 578(20.2)$ & $179 / 743(24.1)$ & 64/225 (28.4) & 123/452 (27.2) \\
\hline 2 & $148 / 578(25.6)$ & $201 / 743(27.1)$ & 60/225 (26.7) & 132/452 (29.2) \\
\hline 3 & $143 / 578(24.7)$ & $168 / 743(22.6)$ & $51 / 225(22.7)$ & 97/452 (21.5) \\
\hline 4 & $156 / 578(27.0)$ & $174 / 743(23.4)$ & $41 / 225(18.2)$ & $83 / 452(18.4)$ \\
\hline$\geq 5$ & $14 / 578(2.4)$ & $21 / 743(2.8)$ & $9 / 225(4.0)$ & $17 / 452(3.8)$ \\
\hline Median & 3 & 2 & 2 & 2 \\
\hline \multicolumn{5}{|l|}{ HPV infection and disease at baseline } \\
\hline $\begin{array}{l}\text { Squamous intraepithelial lesion present on } \\
\text { cytology: }\end{array}$ & $208 / 570(36.5)$ & $225 / 749(30.0)$ & $61 / 226(27.0)$ & $66 / 464(14.2)$ \\
\hline Atypical cells of undetermined significance & $47 / 570(8.2)$ & $65 / 749(8.7)$ & $16 / 226(7.1)$ & $17 / 464(3.7)$ \\
\hline Atypical cells, cannot exclude high grade lesion & $13 / 570(2.3)$ & $10 / 749(1.3)$ & $0 / 226(0.0)$ & $3 / 464(0.6)$ \\
\hline Low grade lesion & $112 / 570(19.6)$ & $120 / 749(16.0)$ & $42 / 226(18.6)$ & $45 / 464(9.7)$ \\
\hline High grade lesion & $36 / 570(6.3)$ & $29 / 749(3.9)$ & $3 / 226(1.3)$ & $1 / 464(0.2)$ \\
\hline Atypical glandular cells & $0 / 570(0.0)$ & $1 / 749(0.1)$ & $0 / 226(0.0)$ & $0 / 464(0.0)$ \\
\hline PCR positive for $\geq 1 \mathrm{HPV}$ type at baselinet: & $410 / 585(70.1)$ & $471 / 760(62.0)$ & $151 / 229(65.9)$ & 208/473 (44.0) \\
\hline Vaccine HPV type: & $254 / 584(43.5)$ & $262 / 759$ (34.5) & 95/228 (41.7) & $112 / 473(23.7)$ \\
\hline HPV6 & $59 / 582(10.1)$ & $60 / 754(8.0)$ & 60/226 (26.5) & $55 / 473(11.6)$ \\
\hline HPV11 & 13/582 (2.2) & $6 / 757(0.8)$ & 6/228 (2.6) & $5 / 473(1.1)$ \\
\hline HPV16 & $183 / 581(31.5)$ & 187/755 (24.8) & 45/228 (19.7) & $52 / 473(11.0)$ \\
\hline HPV18 & $60 / 580(10.3)$ & $57 / 757(7.5)$ & $12 / 228(5.3)$ & $24 / 473(5.1)$ \\
\hline Non-vaccine type: & $333 / 584(57.0)$ & $389 / 758$ (51.3) & $107 / 229(46.7)$ & $165 / 472(35.0)$ \\
\hline HPV31 & 78/577 (13.5) & $96 / 755(12.7)$ & $17 / 228(7.5)$ & $31 / 471(6.6)$ \\
\hline HPV33 & 32/582 (5.5) & $44 / 754(5.8)$ & $5 / 228(2.2)$ & 13/472 (2.8) \\
\hline HPV45 & $32 / 582(5.5)$ & 40/752 (5.3) & $7 / 228(3.1)$ & $13 / 472(2.8)$ \\
\hline HPV52 & 79/581 (13.6) & $102 / 755$ (13.5) & 23/228 (10.1) & $41 / 472(8.7)$ \\
\hline HPV58 & $62 / 581(10.7)$ & $81 / 757(10.7)$ & $10 / 228(4.4)$ & 25/472 (5.3) \\
\hline HPV35 & $39 / 581$ (6.7) & 29/756 (3.8) & 8/229 (3.5) & 9/472 (1.9) \\
\hline HPV39 & $68 / 580(11.7)$ & 71/753 (9.4) & 21/228 (9.2) & $30 / 472(6.4)$ \\
\hline HPV51 & $101 / 581(17.4)$ & $112 / 752(14.9)$ & $34 / 227(15.0)$ & 44/472 (9.3) \\
\hline
\end{tabular}

Women who had diagnosis of vulvar or vaginal disease ${ }^{*}$ Vaccine $(n=229) \quad$ Placebo $(n=475)$

$19.9(1.9) \quad 19.3(2.1)$

$19.9(2.0)$

$19.9(2.0) \quad 19.8(2.0)$

(1)


Table 1 (continued)

\begin{tabular}{|c|c|c|c|c|}
\hline & \multicolumn{2}{|c|}{ Women who had cervical surgery } & \multicolumn{2}{|c|}{ Women who had diagnosis of vulvar or vaginal disease ${ }^{*}$} \\
\hline & Vaccine $(n=587)$ & Placebo $(n=763)$ & Vaccine $(n=229)$ & Placebo $(n=475)$ \\
\hline HPV56 & $105 / 579(18.1)$ & $115 / 753(15.3)$ & $31 / 227(13.7)$ & $39 / 471(8.3)$ \\
\hline HPV59 & $56 / 580(9.7)$ & $66 / 754(8.8)$ & $16 / 228(7.0)$ & $32 / 471(6.8)$ \\
\hline
\end{tabular}

$\mathrm{HPV}=$ human papillomavirus. $\mathrm{PCR}=$ polymerase chain reaction.

${ }^{*}$ Comprises genital warts, vulvar intraepithelial neoplasia, or vaginal intraepithelial neoplasia.

†Positive for $\geq 1$ of the listed HPV types at day 1 on at least 1 required swab or (if obtained) biopsy sample. 
Table 2| Impact of quadrivalent HPV vaccine on incidence of subsequent HPV related disease* among women who had undergone cervical surgery

\begin{tabular}{|c|c|c|c|c|c|}
\hline \multirow[b]{2}{*}{ End point } & \multicolumn{2}{|c|}{ Vaccine $(n=587)$} & \multicolumn{2}{|c|}{ Placebo $(n=763)$} & \multirow[b]{2}{*}{$\begin{array}{l}\% \text { reduction }(95 \% \mathrm{Cl}) \text { in } \\
\text { rate with vaccine }\end{array}$} \\
\hline & $\begin{array}{c}\text { No of women with a } \\
\text { lesiont }\end{array}$ & Rate‡ & $\begin{array}{c}\text { No of women with a } \\
\text { lesiont }\end{array}$ & Rate‡ & \\
\hline \multicolumn{6}{|c|}{ HPV related disease irrespective of causal HPV type } \\
\hline Any disease: & $45 / 475$ & 6.6 & $94 / 593$ & 12.2 & 46.2 (22.5 to 63.2$)$ \\
\hline $\begin{array}{l}\text { Cervical intraepithelial neoplasia } \\
\text { grade I or worse }\end{array}$ & $30 / 474$ & 4.3 & $65 / 592$ & 8.2 & $48.3(19.1$ to 67.6$)$ \\
\hline Genital warts & $7 / 474$ & 1.0 & 22/589 & 2.6 & $63.0(10.3$ to 86.6$)$ \\
\hline $\begin{array}{l}\text { Vulvar or vaginal intraepithelial } \\
\text { neoplasia grade I or worse }\end{array}$ & $12 / 474$ & 1.7 & 19/589 & 2.3 & $26.5(-59.5$ to 67.5$)$ \\
\hline $\begin{array}{l}\text { Cervical intraepithelial neoplasia } \\
\text { grade II or worse }\end{array}$ & $8 / 474$ & 1.1 & 26/592 & 3.1 & 64.9 (20.1 to 86.3$)$ \\
\hline $\begin{array}{l}\text { Cervical intraepithelial neoplasia } \\
\text { grade III or worse }\end{array}$ & $3 / 474$ & 0.4 & $13 / 592$ & 1.5 & 73.5 (3.4 to 95.2$)$ \\
\hline $\begin{array}{l}\text { Vulvar or vaginal intraepithelial } \\
\text { neoplasia grade II or worse }\end{array}$ & $3 / 474$ & 0.4 & $5 / 589$ & 0.6 & 30.1 ( -259.1 to 89.2$)$ \\
\hline \multicolumn{6}{|c|}{ Disease related to vaccine HPV types $(6,11,16$, or 18$)$} \\
\hline Any disease: & $6 / 475$ & 0.8 & $33 / 593$ & 3.9 & 79.1 (49.4 to 92.8$)$ \\
\hline $\begin{array}{l}\text { Cervical intraepithelial neoplasia } \\
\text { grade I or worse }\end{array}$ & $2 / 474$ & 0.3 & $9 / 592$ & 1.1 & $74.2(-24.8$ to 97.3$)$ \\
\hline Genital warts & $2 / 474$ & 0.3 & $21 / 589$ & 2.5 & 89.0 (54.9 to 98.7$)$ \\
\hline $\begin{array}{l}\text { Vulvar or vaginal intraepithelial } \\
\text { neoplasia grade I or worse }\end{array}$ & $2 / 474$ & 0.3 & $6 / 589$ & 0.7 & $61.3(-116.7$ to 96.2$)$ \\
\hline $\begin{array}{l}\text { Cervical intraepithelial neoplasia } \\
\text { grade II or worse }\end{array}$ & $1 / 474$ & 0.1 & $3 / 592$ & 0.4 & $61.3(-382.4$ to 99.3$)$ \\
\hline $\begin{array}{l}\text { Cervical intraepithelial neoplasia } \\
\text { grade III or worse }\end{array}$ & $0 / 474$ & 0.0 & $0 / 592$ & 0.0 & NA \\
\hline $\begin{array}{l}\text { Vulvar or vaginal intraepithelial } \\
\text { neoplasia grade II or worse }\end{array}$ & $1 / 474$ & 0.1 & $3 / 589$ & 0.4 & $61.2(-383.1$ to 99.3$)$ \\
\hline
\end{tabular}

HPV=human papillomavirus.

*Any HPV related disease detected $\geq 60$ days after surgery.

†Number of women with at least one follow-up visit for the respective end point after surgery. A woman is counted only once for each end point (that is, once in each row) but may have developed more than one end point (and so may appear in more than one row).

$\ddagger$ Cases per 100 person years at risk. 
Table 3| Impact of quadrivalent HPV vaccine on incidence of subsequent cervical intraepithelial neoplasia grade I or worse* associated with 10 tested non-vaccine HPV types among women who had undergone cervical surgery

\begin{tabular}{|c|c|c|c|c|c|}
\hline \multirow[b]{2}{*}{ HPV type } & \multicolumn{2}{|c|}{ Vaccine $(n=587)$} & \multicolumn{2}{|c|}{ Placebo $(n=763)$} & \multirow[b]{2}{*}{$\begin{array}{l}\text { \% reduction }(95 \% \mathrm{Cl}) \text { in rate } \\
\text { with vaccine }\end{array}$} \\
\hline & $\begin{array}{c}\text { No of women with a } \\
\text { lesiont }\end{array}$ & Rate‡ & $\begin{array}{c}\text { No of women with a } \\
\text { lesiont }\end{array}$ & Rate‡ & \\
\hline $\begin{array}{l}\text { Any tested non-vaccine } \\
\text { type: }\end{array}$ & $17 / 474$ & 2.4 & $34 / 592$ & 4.1 & $42.5(-5.9$ to 69.8$)$ \\
\hline $\begin{array}{l}\text { HPV types } 31,33,45 \text {, } \\
52, \text { or } 58\end{array}$ & $9 / 474$ & 1.2 & $24 / 592$ & 2.9 & 56.6 (3.4 to 82.3 ) \\
\hline HPV31 & $1 / 474$ & 0.1 & $10 / 592$ & 1.2 & 88.4 (18.6 to 99.7$)$ \\
\hline HPV33 & $2 / 474$ & 0.3 & $3 / 592$ & 0.3 & 22.1 (-579.7 to 93.5$)$ \\
\hline HPV35 & $3 / 474$ & 0.4 & 2/592 & 0.2 & $-76.3(-2010.8$ to 79.8$)$ \\
\hline HPV39 & $3 / 474$ & 0.4 & $3 / 592$ & 0.4 & $-16.8(-772.3$ to 84.4$)$ \\
\hline HPV45 & $0 / 474$ & 0.0 & $4 / 592$ & 0.5 & $100.0(-76.2$ to 100.0$)$ \\
\hline HPV51 & $1 / 474$ & 0.1 & $7 / 592$ & 0.8 & 83.4 (-29.1 to 99.6$)$ \\
\hline HPV52 & $5 / 474$ & 0.7 & 10/592 & 1.2 & $41.7(-87.1$ to 84.4$)$ \\
\hline HPV56 & $3 / 474$ & 0.4 & $11 / 592$ & 1.3 & $68.2(-20.2$ to 94.3$)$ \\
\hline HPV58 & $1 / 474$ & 0.1 & $5 / 592$ & 0.6 & $76.6(-108.9$ to 99.5$)$ \\
\hline HPV59 & $1 / 474$ & 0.1 & $3 / 592$ & 0.3 & 61.1 (-384.9 to 99.3$)$ \\
\hline
\end{tabular}

HPV=human papillomavirus.

${ }^{*}$ Cervical intraepithelial neoplasia detected $\geq 60$ days after surgery.

†Number of women with at least one follow-up visit for the respective end point after surgery. A woman is counted only once for each end point (that is, once in each row) but may have developed more than one end point (and so may appear in more than one row).

$\ddagger$ Cases per 100 person years at risk. 
Table 4| Impact of quadrivalent HPV vaccine on incidence of subsequent HPV related disease among women who had a diagnosis of vulvar or vaginal disease*

\begin{tabular}{|c|c|c|c|c|c|}
\hline \multirow[b]{2}{*}{ End point } & \multicolumn{2}{|c|}{ Vaccine $(n=229)$} & \multicolumn{2}{|c|}{ Placebo $(n=475)$} & \multirow[b]{2}{*}{$\begin{array}{l}\% \text { reduction }(95 \% \mathrm{Cl}) \text { in } \\
\text { rate with vaccine }\end{array}$} \\
\hline & $\begin{array}{c}\text { No of women with a } \\
\text { lesion† }\end{array}$ & Rate‡ & $\begin{array}{c}\text { No of women with a } \\
\text { lesion† }\end{array}$ & Rate‡ & \\
\hline \multicolumn{6}{|c|}{ HPV related disease irrespective of causal HPV type } \\
\hline Any disease: & $70 / 211$ & 20.1 & $163 / 422$ & 31.0 & $35.2(13.8$ to 51.8$)$ \\
\hline $\begin{array}{l}\text { Cervical intraepithelial neoplasia } \\
\text { grade I or worse }\end{array}$ & $39 / 210$ & 9.8 & $110 / 421$ & 18.2 & 46.3 (22.0 to 63.7$)$ \\
\hline Genital warts & $19 / 209$ & 4.7 & $47 / 413$ & 7.2 & $35.6(-11.9$ to 64.3$)$ \\
\hline $\begin{array}{l}\text { Vulvar or vaginal intraepithelial } \\
\text { neoplasia grade I or worse }\end{array}$ & $28 / 209$ & 6.9 & $54 / 413$ & 8.4 & $18.4(-31.1$ to 50.3$)$ \\
\hline $\begin{array}{l}\text { Cervical intraepithelial neoplasia } \\
\text { grade II or worse }\end{array}$ & $13 / 210$ & 3.0 & $35 / 421$ & 5.1 & $40.8(-14.6$ to 71.3$)$ \\
\hline $\begin{array}{l}\text { Cervical intraepithelial neoplasia } \\
\text { grade III or worse }\end{array}$ & $10 / 210$ & 2.3 & $20 / 421$ & 2.8 & $18.5(-82.5$ to 65.9$)$ \\
\hline $\begin{array}{l}\text { Vulvar or vaginal intraepithelial } \\
\text { neoplasia grade II or worse }\end{array}$ & $11 / 209$ & 2.6 & $23 / 413$ & 3.4 & $23.5(-63.3$ to 66.4$)$ \\
\hline \multicolumn{6}{|c|}{ Disease related to vaccine HPV types $(6,11,16$, or 18$)$} \\
\hline Any disease: & $20 / 211$ & 4.9 & $84 / 422$ & 13.8 & $64.4(41.6$ to 79.3$)$ \\
\hline $\begin{array}{l}\text { Cervical intraepithelial neoplasia } \\
\text { grade I or worse }\end{array}$ & $8 / 210$ & 1.9 & $44 / 421$ & 6.6 & 71.8 (39.5 to 88.5$)$ \\
\hline Genital warts & $10 / 209$ & 2.4 & $39 / 413$ & 5.9 & $60.4(19.1$ to 82.3$)$ \\
\hline $\begin{array}{l}\text { Vulvar or vaginal intraepithelial } \\
\text { neoplasia grade I or worse }\end{array}$ & $6 / 209$ & 1.4 & $21 / 413$ & 3.1 & $54.5(-16.5$ to 85.0$)$ \\
\hline $\begin{array}{l}\text { Cervical intraepithelial neoplasia } \\
\text { grade II or worse }\end{array}$ & $5 / 210$ & 1.1 & $19 / 421$ & 2.7 & $57.7(-17.0$ to 87.7$)$ \\
\hline $\begin{array}{l}\text { Cervical intraepithelial neoplasia } \\
\text { grade III or worse }\end{array}$ & $5 / 210$ & 1.1 & $11 / 421$ & 1.5 & $25.8(-131.8$ to 79.8$)$ \\
\hline $\begin{array}{l}\text { Vulvar or vaginal intraepithelial } \\
\text { neoplasia grade II or worse }\end{array}$ & $3 / 209$ & 0.7 & $13 / 413$ & 1.9 & $63.0(-34.8$ to 93.2$)$ \\
\hline
\end{tabular}

HPV=human papillomavirus.

*Any HPV related disease detected $\geq 60$ days after diagnosis of genital warts, vulvar intraepithelial neoplasia, or vaginal intraepithelial neoplasia.

†Number of women with at least one follow-up visit for the respective end point after surgery. A woman is counted only once for each end point (that is, once in each row) but may have developed more than one end point (and so may appear in more than one row).

$\ddagger$ Cases per 100 person years at risk. 


\section{Figures}

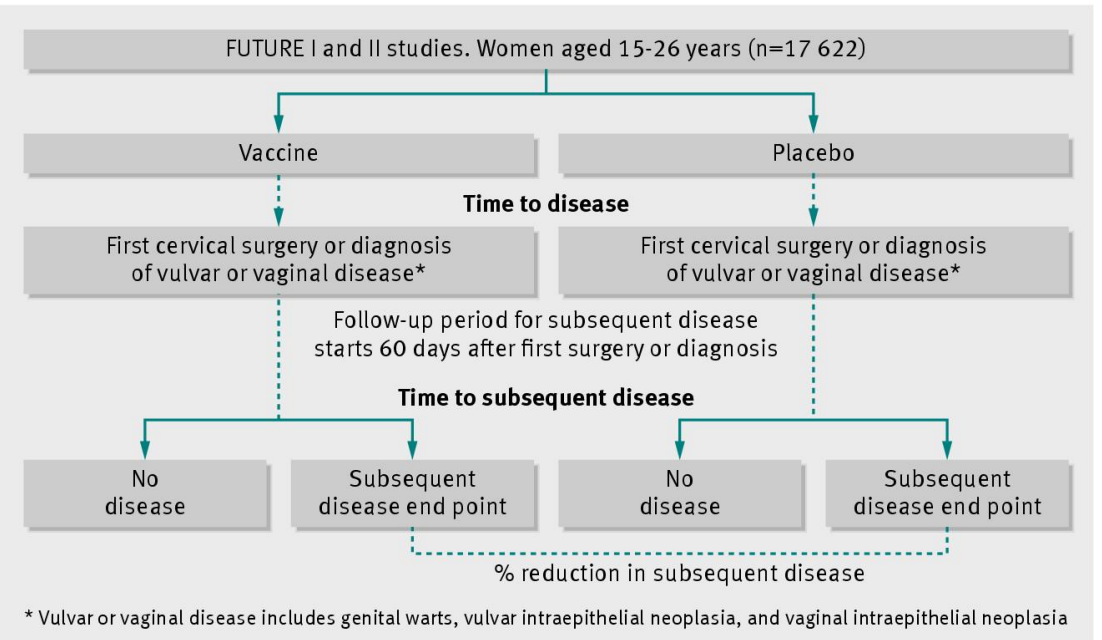

Fig 1 Study design for assessing effect of quadrivalent HPV vaccine on incidence of subsequent HPV related disease among women who had undergone surgery for cervical disease or who were diagnosed with vulvar or vaginal disease. Subsequent disease was measured from 60 days after surgery or diagnosis

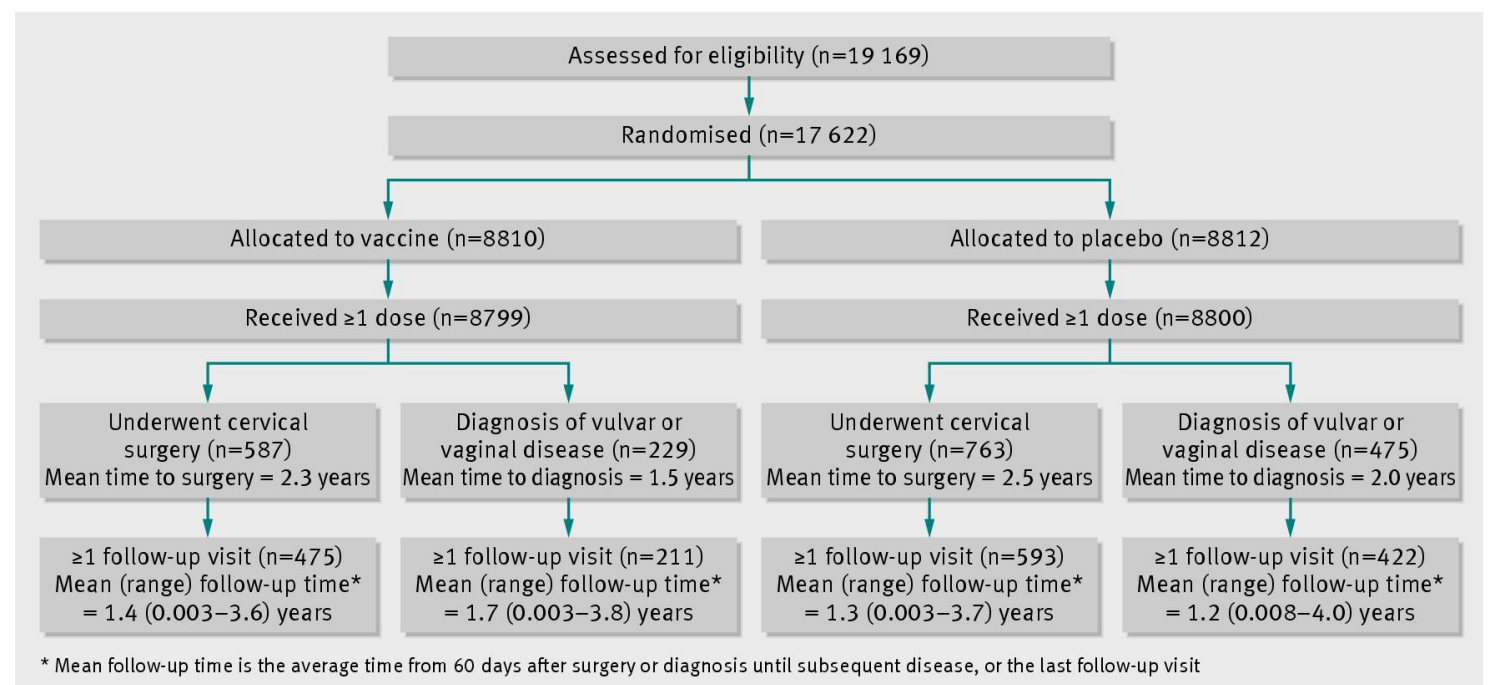

Fig 2 Participant flow through study 


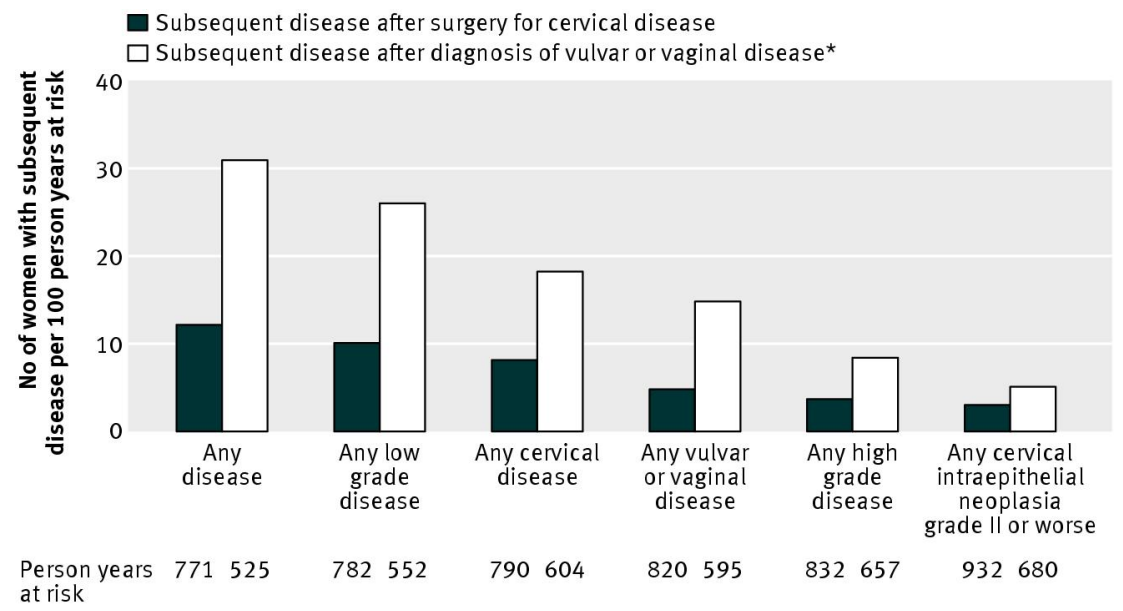

*Comprises genital warts, vulvar intraepithelial neoplasia, and vaginal intraepithelial neoplasia

Fig 3 Incidence of HPV related disease detected $\geq 60$ days after cervical surgery or diagnosis of vulvar or vaginal disease among women who did not receive quadrivalent HPV vaccine (that is, placebo recipients)

- Quadrivalent vaccine --- Placebo

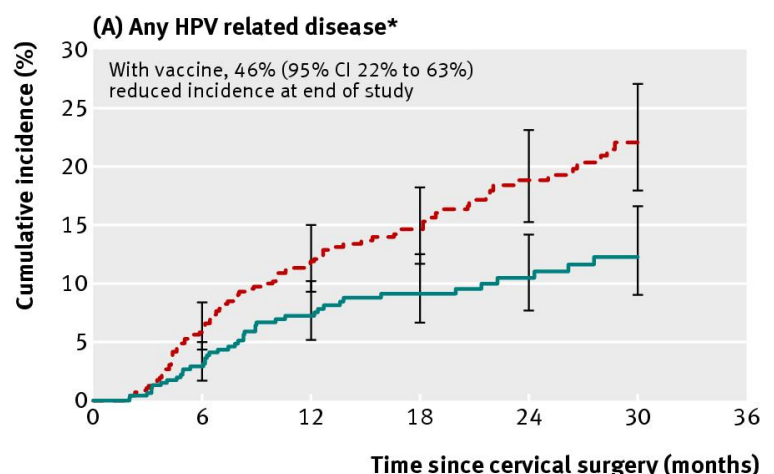

(C) Any HPV related disease*

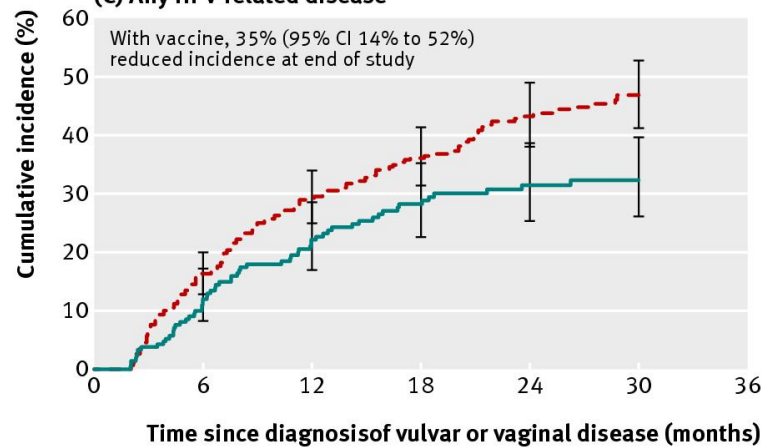

(B) Vulvar or vaginal diseaset

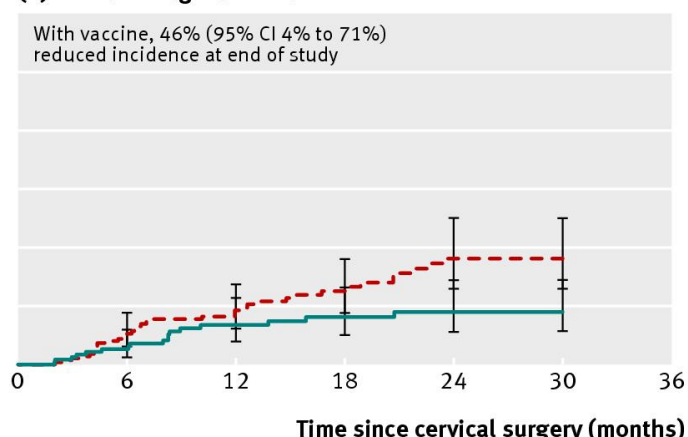

(D) Any cervical diseaseł

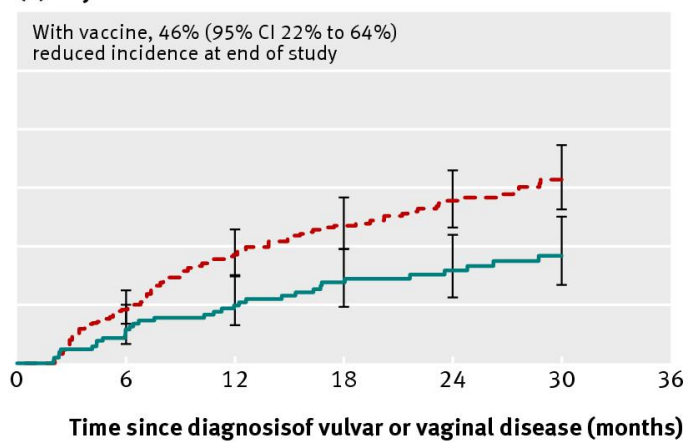

*Cervical intraepithelial grade I or worse, genital warts, vulvar intraepithelial neoplasia grade I or worse, or vaginal intraepithelial neoplasia grade I or worse tGenital warts, vulvar intraepithelial neoplasia grade I or worse, or vaginal intraepithelial neoplasia grade I or worse \#Cervical intraepithelial grade I or worse

Fig 4 Time to detection of any HPV related disease (A) or vulvar or vaginal disease (B) after cervical surgery; and of any HPV related disease (C) or any cervical disease (D) after diagnosis of vulvar or vaginal disease. Case counting began 60 days after surgery or diagnosis 